\section{Medical Errors, Adverse Medical Events, and PDRM}

A MEDLINE search in August 2002 using the search term "PDRM" revealed 2 medical literature citations, one on the subject of ventilator-associated pneumonia and potentially drugresistant microorganisms (PDRM) ${ }^{1}$ and the other an overview of the contribution of systems to the "widespread problem of preventable drug-related morbidity." Expanding the search to the terms "drug-related morbidity" yielded 667 citations, including the widely referenced article on a cost-of-illness model for drugrelated morbidity and mortality by Johnson and Bootman in 1995. ${ }^{3}$

As MacKinnon and Hepler describe in this issue of the Journal, ${ }^{4}$ the distinction between drug-related morbidity (DRM) and preventable DRM (PDRM) is obviously of importance to managed care pharmacists since PDRM would, by definition, be reducible. Their research suggested that experts can agree on what constitutes a preventable drug-related adverse event. How does this research advance our work in making medication delivery systems and drug therapy management safer for its users? The results of the PDRM work to define a nomenclature and measurement system in a hospital health care system, among the elderly, will be described in an article in the November/December 2002 issue of JMCP. Their expert panel reached consensus on several clinical indicators of PDRM that included indicator no. 6, an emergency room (ER) visit or hospitalization due to hyperkalemia subsequent to the use of an ACE (angiotensin-converting enzyme) inhibitor without checking electrolytes and $\mathrm{CBC}$ at least every 6 months. By this measure, most of our elderly population on an ACE inhibitor could be at risk of PDRM.

The work by Morris and Cantrill in the United Kingdom to validate and apply the U.S.-derived PDRM indicators developed by MacKinnon and Hepler resulted in survival of just 19 (33\%) of the 57 PDRM indicators in the U.K. ${ }^{5}$ This result suggests that the U.S.-derived PDRM indicators are either (a) not durable under scrutiny for validity and relevance in general or (b) quality indicators in drug therapy management are significantly different in the U.K. compared to the U.S. In either case, the fact remains that these expert panels, small in the number of experts employed, 7 in the U.S. and 16 in the U.K, did not agree on 2 out of 3 PDRM indicators.

Already, much value has been derived from the distinction between medical error (ME) and adverse event (AE), the former referring to any event that represents a mistake versus the latter that is associated with an adverse outcome. ${ }^{6}$ Whether an adverse outcome represents preventable "morbidity" is an important matter for investigators and those dedicated to quality improvement. Administering the wrong drug (aspirin) to a patient with a known aspirin allergy and prescribed acetaminophen represents an apparent medical error. This ME may have no adverse effect and therefore not represent an AE. Without an adverse effect, there is no opportunity for a preventable drug-related adverse event and no morbidity to be prevented. Had the patient suffered anaphylactic shock due to an aspirin allergy and died, the event would presumably represent an $\mathrm{ME}$, an $\mathrm{AE}$, a drug-related event, an adverse drug event, an adverse drug reaction, and preventable drug-related mortality, but not PDRM.

Fortunately for patients and users of the U.S. health care system, the focus of promoting patient safety and preventing medical error shifted years ago to systems and away from people. More than 10 years ago, Reason summarized his work in the analysis of success and failure in complex work settings: "Rather than being the main instigators of an accident, operators tend to be the inheritors of system defects ... . Their part is that of adding the final garnish to a lethal brew whose ingredients have already been long in the cooking." "In 1997-1998, the Joint Commission on Accreditation of Healthcare Organizations (JCAHO) revised its policy on reporting medical errors to emphasize the investigation of sources of medical errors and self-correction versus the former policy of public notice and punishment through threat of downgrade of accreditation status. ${ }^{9}$ More recently, JCAHO standards for medical error reporting, adopted in mid-2001, included further emphasis on process-oriented investigation by shifting the focus from external review and reporting to development of a system within each hospital (system) to report and analyze medical errors. ${ }^{10}$

\section{Crossing the Quality Chasm-Incremental Change through Clinical Practice Guidelines (CPGs)}

The explosion of data available on the Internet has magnified the challenge of filtering that data to derive information. Information is that precious commodity that, unlike data, supports action that is more likely to result in desired outcomes. In addition to the mountain of data available in the lay press, Internet, television, and radio, the U.S. National Library of Medicine reported an average 10,000 new lines (articles) referenced in MEDLINE each week at year-end 2001. ${ }^{11}$ How can we possibly separate the reliable and useful information from the overwhelming amount of data available today?

We appear to be approaching a time when information technology has the potential to eliminate quality shortfalls in certain sectors of the U.S. health care system. ${ }^{12}$ Yet, we still have a long way to go, particularly in the creation of an electronic medical record that will support fully informed decision making by health care providers and patients, at the point of care. Lurking behind the obvious challenges is the never-ending task of developing and maintaining clinical practice guidelines that can help reduce nonrandom, systematic variation in medical interventions and delivery of pharmacy services. Despite all of the challenges, the use of CPGs or clinical practice models (CPMs) is the foundation for incremental change that will permit improvements in systems to better protect patient safety and improve clinical and service outcomes.

Many in health care refer to "evidence-based medicine" as if this concept exists commonly in practice. It would be comforting and helpful if reliable information (evidence) was routinely reduced to useful guidelines and available in a readily accessible manner, at the point of care. Work published in September 2001 found that only 3 of 17 (18\%) of the CPGs published by 
the Agency for Healthcare Research and Quality were judged to still be valid. ${ }^{13}$ Seven (41\%) of the CPGs were found to require a major update to reflect new evidence, and 6 CPGs were judged to require a "minor update." In other words, at the time of the report in September 2001, three quarters of the AHRQ guidelines needed updating.

In fact, there are at least 8 threats to the reliability and usefulness of information contained in CPGs and CPMs. First, bias and conflict of interest can undermine validity and reliability (value) of the clinical "evidence." Second, experts may fail to use sufficient rigor when interpreting the results of clinical interventions. Third, valid and reliable clinical evidence may not be reduced to a practical and useful CPG. Fourth, experts may not disclose conflict of interest and potential bias when preparing CPGs. Fifth and sixth, a useful and practical CPG may not be disseminated adequately or may be unavailable at the point of care. Seventh, the communication of the guideline may be ineffective or become lost in the increasing level of "noise" in information communicated to physicians. Eighth, even if readily available at the point of care, the clinician may reject or otherwise choose to ignore the $\mathrm{CPG}$ and the information it contains. All 8 barriers may contribute to nonrandom variation in clinical practice.

An online survey of about 300 internal medicine and family practice physicians conducted by Harris Interactive in mid-2001 for the Henry J. Kaiser Family Foundation found that only 19\% of these primary care physicians chose aspirin as the treatment of choice for the onset of stroke, $27 \%$ chose heparin, and about half opted for t-PA. According to clinical evidence, t-PA is the thirdline choice for acute stroke, while aspirin is first-line and heparin is second-line. Some of the gap in the use of CPGs is attributable to physician resistance to "cookbook medicine." Alan Muney, MD, chief medical officer for Oxford Health Plans (Trumbull, Connecticut) commented, "The shameful truth is that not all doctors adhere to evidence-based medical guidelines."14 "Shameful" might not be the best word to describe the failure by clinicians to use the available information since practicing clinicians point, fairly, to bias in the preparation of many CPGs and CPMs. There is also the matter of inadequate communication of the CPG to the practitioner. Amid an increasing barrage of data, practitioners could easily lose the significance of an otherwise important clinical guideline among the "noise" of data overload.

The matter of pseudo-evidence is also of serious concern and contributes to the problem of data overload and information underload. Calling data "evidence" does not make it so. Much of the literature contains expert opinion, not the results of randomized clinical trials. It has been said that one study, particularly a randomized, control group trial, is worth 1,000 expert opinions.

\section{Information Technology to Cross the Quality Chasm}

At year-end 1997, the Health Care Financing Administration, now the Centers for Medicare and Medicaid Services, proposed a maximum 2\% medication error rate for Medicare and Medicaid hospitals, and nurses would be required "to review drug orders for accuracy of the entire system before prescription drugs are administered."15 In 1998, a 15-month study of computerized physician-order entry at Brigham \& Women's Hospital found that medication errors were reduced by $55 \%$ when physicians were required to enter all drug orders by computer. ${ }^{16}$ While few would debate the need to improve quality by reducing medication error rates, there is debate about the magnitude of the problem.

More than 2 years later, General Motors Corp., IBM, AT\&T, General Electric, Boeing, and 91 other employers collaborated to form the Leapfrog Group, to, as its name implies, leapfrog over the present (slow) pace of quality improvement in health care. The Leapfrog Group in 2001 defined 3 basic ways to improve safety and quality of health care for at least the hospital component of the health system. The Leapfrog Group urged managed care organizations (MCOs) to contract with only those hospitals that (1) implemented a computerized order entry system by 2004 and (2) provided an ICU staffed full-time by an internist with specialty training and certification in intensive care medicine. Third, the Leapfrog Group suggested that MCOs should practice evidence-based medicine by sending patients only to hospitals with high volume and favorable outcomes. As for the chasm between current practice and the 3 quality standards: only $3.3 \%$ of hospitals reported operational physician electronic order entry systems, only $10 \%$ of hospitals surveyed had intensive-care specialists overseeing care in the ICU at least 8 hours per day, and only $12 \%$ of 250 hospitals surveyed by the Leapfrog Group in late 2001 met the standard of performing at least 500 coronary artery bypass graft procedures per year.

At a meeting on July 26, 2002, called by the U.S. Food and Drug Administration (FDA), hospital groups and patient safety advocates such as the Institute for Safe Medication Practices (ISMP) asked the FDA to require prescription drug manufacturers to apply bar codes to all drug packages, particularly unitdose packages. The promise is large for information technology (IT) to reduce the estimated 100,000 deaths annually and the estimated 770,000 medication errors that occur in U.S. hospitals each year. ${ }^{17}$ Yet, there are several factors that should affect expectations of the promise of IT to cross the quality chasm in medication errors. First, there is the absence of a single bar code standard. Second, even if there were bar codes on individual drug doses (unit dose packages), only 10\% to 15\% of hospitals had bar code readers at patient bedsides in mid-2002. Third, drug manufacturers had been producing fewer drugs in unitdose packages, due to the extra cost. Fourth, there is a question of the return-on-investment. Bar coding drugs and making bar code readers standard at hospital bedsides would cost $\$ 1.5$ billion or more, and the FDA estimate of the 770,000 annual hospital medication errors that are preventable is a wide range, from $28 \%$ to $95 \% .^{18}$ 
Lucian Leape, MD, a retired surgeon and professor at the Harvard School of Public Health, found wide discrepancies in hospital medication error rates, attributing the discrepancies to the method of data reporting rather than actual variation in incidence and outcomes, highlighting the fact that people who report high medication error rates are punished for these data. Leape found an error rate of $0.2 \%$ (2 per 1,000 charts) for selfreport methods, $0.7 \%$ for retrospective chart reviews, $3.8 \%$ for computer screening, $6.5 \%$ for daily chart reviews, and $10.0 \%$ for computer screening combined with daily chart review. He concluded, "If you are relying on incident reports, you are missing $95 \%$ of them."

So, it would seem that (a) everybody agrees that medical errors should not occur, (b) the scope of the problem is not well-defined quantitatively, and (c) we have solutions for reducing medication errors but determining the return-on-investment from these solutions will be difficult. Brent James, MD, a well-recognized leader and teacher in health care quality improvement, has observed for several years that the key to reducing systematic variation in health care delivery involves "making it easy to do it right." This perspective involves making key process steps routine and unavoidable, such as the application of IT to the hospital discharge process. A randomized trial of a computerized clinical-information system that generated preventive care reminders at the point of patient discharge increased significantly the use of subcutaneous heparin for patients at risk for venous thromboembolism, instructions to take aspirin for patients hospitalized for acute myocardial infarction, and the use of pneumococcal or influenza vaccine for eligible patients. ${ }^{19}$ James observed that "studies of clinical errors have identified a series of human limitations that lead to predictable failures of the health care delivery system. ${ }^{.20}$ In this issue of the Journal, James elaborates on his perspectives of the quality chasm in health care. ${ }^{21}$ His perspectives are important since his work on the Institute of Medicine "Chasm" report is almost incidental to his efforts over the last 20 years to improve health care delivery by teaching others the methods to apply analytical thinking and statistical tools to the study of the processes of care, measuring outcomes, and reducing nonrandom variation in clinical practice.

\section{Benefit Maximums Versus Drug Benefit Needs for Medicare Beneficiaries}

Annual dollar maximum limits for Medicare+Choice prescription drug benefits are common, an average \$1,149 in 1997 and as low as $\$ 600$ per year. By CY 2000, 38\% of Medicare+Choice members with prescription drug benefits had an annual maximum of $\$ 750$ or less. Data from the Kaiser Family Foundation also show that $13 \%$ of Medicare beneficiaries spent $\$ 2,000$ or more on prescription drugs in CY 2001, accounting for 52\% of total prescription drug spending for Medicare beneficiaries. ${ }^{22}$ Spending of $\$ 1,000$ or more was found among $28 \%$ of Medicare beneficiaries and accounted for $76 \%$ of total expenditures for prescription drugs. Yet, an amazing $17 \%$ of Medicare beneficiaries had no (\$0) spending on prescription drugs in CY 2001. A previous study in the Journal reported that for 2 cohorts of patients in a Medicare+Choice population, one using only community pharmacy services and the other using only mail-service pharmacy, only $0.18 \%$ (26 members) and $0.01 \%$ (2 members) respectively, reached their maximum drug benefi, which ranged from $\$ 500$ to $\$ 1,600$ in $1998 .^{23}$ These data are difficult to reconcile.

Survey data from 10,927 noninstitutionalized seniors in 8 geographically diverse states in 2001 showed that $35 \%$ of seniors with drug coverage under a Medigap policy, $25 \%$ of seniors enrolled in state pharmacy assistance programs, and 19\% of seniors in Medicare HMOs spent at least $\$ 100$ per month $(\$ 1,200$ per year) on prescriptions in 2001. ${ }^{24}$ Medicare HMOs were important sources of drug coverage for seniors in California (30\%) and Colorado (24\%) but were less important in other states, ranging from a low of $7 \%$ in Illinois to $14 \%$ in Pennsylvania.

In this issue, Cox and Henderson ${ }^{25}$ report that 24\% of the respondents in their survey of Medicare+Choice members (with limited prescription drug benefits) did not know if they had exceeded their annual drug benefit maximum in the preceding year (CY 2000), and 46\% did not know (accurately) the dollar amount of their annual benefit maximum ("cap"). This study did not link the survey responses to the demographics and drug benefits of the Medicare+Choice members. This link would have helped answer questions that arise from efforts to interpret the survey results. And, the fact that $24 \%$ of Medicare+Choice members reported that they were not aware of whether or not they had exhausted their prescription drug benefits may not be surprising in the context of the statistic that $17 \%$ of Medicare beneficiaries had no $(\$ 0)$ expenditures for prescription drugs in CY 2001.

The study by Cox and Henderson also did not include analysis of the reported behaviors compared to actual experience, as measured by drug claims in the database. This assessment would have provided more insight into this important question of the effects of Medicare+Choice annual maximum benefits on the use of prescription drugs by type (e.g., generic drugs versus brand drugs, antihypertensives versus drugs for heartburn). Also left to other researchers is evaluation of the qualitative and quantitative time curve to exhaustion of benefits; i.e., the percentage of beneficiaries who exhaust benefits in 90 days, 120 days, etc. Readers should also note that the work by Cox and Henderson did not include Medicare+Choice annual maximum benefits applied quarterly (e.g., \$1,000 annual maximum limited to $\$ 250$ per calendar quarter), a common practice in Medicare+Choice plans since 2001.

Nevertheless, the study by Cox and Henderson does highlight a subject that should concern health plan administrators 
and pharmacy benefit mangers, that is, the adequacy of pharmacy benefits and the potential consequences that could arise from gaps in treatment associated with financial barriers. A Kaiser Family Foundation study published in 2002 found that 30\% of the nonelderly uninsured did not fill a prescription in the previous 12 months due to cost, compared to $12 \%$ of the nonelderly with insurance, ${ }^{26}$ and $10 \%$ of the insured population reported needing a prescription in the previous 12 months but not getting it due to cost. ${ }^{27}$ Overall, nearly one quarter of seniors, regardless of drug benefit coverage, either did not fill a prescription or skipped doses due to costs. More than one third of seniors without drug coverage either skipped doses or did not fill a prescription due to costs, twice the rate of seniors with drug benefit coverage. ${ }^{24}$

Perhaps the most important finding by Cox and Henderson is the apparent link between Medicare prescription drug benefit maximums and the use of prescription drug samples as a means to mitigate out-of-pocket expenditures. This practice is potentially self-defeating since (a) higher-cost drugs are more heavily sampled and (b) the availability of drug samples may affect physician prescribing practices and reduce the pressure to find lower-cost therapeutic alternatives. Other investigators may ask Medicare+Choice beneficiaries with drug benefit limits the important questions regarding the nature and usefulness of interactions with physicians in offering recommendations for generic drugs and other lower-cost therapeutic alternatives to help reduce out-of-pocket expenditures.

\section{Crossing the Quality Chasm- Pharmacist Prescribing, Nontraditional Interventions, and Outcomes-based Pharmacist Reimbursement (OBPR)}

Since 1996, pharmacists in Indiana have had the authority to manage the drug therapy of hospital patients, including prescribing drugs, when conducted via a protocol established with a physician and a hospital. ${ }^{28}$ In California, AB 826 was enacted late in 2001 and became effective January 1, 2002. AB 826 included 2 key changes in California law: (1) pharmacists may perform clinical functions outside of a pharmacy or other licensed health facility (e.g., the patient's home, physician's office, or medical office building) and (2) pharmacists may select initial drug therapy. Prior law only allowed adjustment of drug therapy (e.g., dose) in outpatient settings. Pharmacist prescriptive authority is permitted only under appropriate protocols, and the pharmacist must notify the prescriber within 24 hours of initiating a drug regimen under this new authority. ${ }^{29}$

By 2002, 33 states granted prescriptive authority to pharmacists, ${ }^{30}$ typically permitting pharmacists to work collaboratively with physicians to adjust drug therapy under protocol. ${ }^{31}$ The collaborative practice agreement with a physician(s) may specify authority for the pharmacist to adjust the dose of prescribed drugs, or in many situations, select initial drug therapy as well. In 1999, Minnesota pharmacists could establish collab- orative practice agreements with physicians allowing pharmacists to adjust drug therapy and administer the first dose in an emergency. The amendments to the pharmacy practice act also recognized the pharmacist's role in counseling, monitoring, and drug research. ${ }^{32}$ In 2002, Maryland HB781 allowed a licensed physician and a licensed pharmacist to enter into a drug therapy management contract. ${ }^{33}$ As defined in the Maryland bill, the contract is a voluntary written arrangement that is disease-state-specific and applies to one pharmacist, one physician, and one patient receiving care from the physician and pharmacist. Connecticut SB528, also enacted in 2002, allowed one or more licensed pharmacists employed by a hospital to enter into a written protocolbased collaborative drug therapy management agreement with one or more physicians to manage the drug therapy of individual patients receiving inpatient services in a hospital. ${ }^{34}$

The need for collaborative work among physicians and pharmacists to reduce medication errors is well-recognized in health systems devoted to quality improvement principles. These organizations are more likely to spawn solutions that recognize the strengths of pharmacists in knowledge of drug dosing and even drug selection. At the University of Wisconsin Hospitals and Clinics, pharmacists have (veto) authority over the prescribing of physicians, most of whom on the house staff are interns and residents. A trial program begun in the trauma unit in 1996 was expanded to a hospital-wide program in 2002, first for all anti-infective orders and later for other therapeutic categories. ${ }^{35}$ Pharmacist oversight of drug orders at the University of Wisconsin Hospitals and Clinics is directed by the hospital's pharmacy and therapeutics committee and will be supplemented in the future by information technology, a computerized physician order entry system.

Recent reports of prescriptive authority for pharmacists and clinical pharmacy interventions in nontraditional roles include a telephone clinic and protocol for eradication of Helicobacter pylori infection. The intervention was associated with favorable outcomes: (a) 100\% patient compliance with HP eradication therapy, (b) symptom improvement in 33\% of patients (but many patients were either asymptomatic or mildly symptomatic before initiating HP eradication therapy), (c) no drug-drug or drug-food interactions, (d) no reinitiation of acid-suppressive therapy at one-month post-treatment, (e) creation of a learning environment for doctor of pharmacy clerkship students in defining a new role for pharmacists in ambulatory care, and (f) pharmacy collaboration with the departments of surgery, medicine, and nursing. ${ }^{36}$

The entire process of quality improvement in health care is based upon incremental change. While the Leapfrog Group encourages us to think of dramatic, fundamental change, the reality is less cataclysmic. As with most industrial and engineering processes, improving the tools improves the quality of the product but only with changes in processes by which the 
tools are used. In drug therapy management, we need the operators (pharmacists) of the tools to embrace clinical practice guidelines and the principles of continuous quality improvement. Compensating pharmacists at the lowest possible price does not foster this attention and commitment.

Farris, Kumbera, Halterman, and Fang in this issue of the Journal, describe a proprietary program developed around the concept of compensating pharmacists based upon measurable patient outcomes. ${ }^{37}$ This is the holy grail of managed care pharmacy, compensation for clinical pharmacists based upon valued outcomes.

\section{Statistical Significance Versus Practical Significance}

Researchers know the value of sample size in demonstrating statistical significance between groups. Small sample size requires a large absolute difference between groups to demonstrate statistical significance. For example, the data used to obtain FDA approval of pravastatin for the indication of stroke involved a study of 9,014 patients with a history of myocardial infarction or unstable angina and total cholesterol levels of 155 to $271 \mathrm{mg}$ per deciliter. The study found that the risk of stroke was 3.7\% among patients given pravastatin versus $4.5 \%$ among patients given placebo over a follow-up period of 6 years, an absolute difference of $0.8 \%$ and relative risk reduction for stroke of $19 \% .{ }^{38}$

Subsequent scrutiny of the study findings and the literature citations identified flaws. Of the 4 studies cited by the authors to support the reported reductions of $25 \%$ to $30 \%$ in the rate of stroke, the West of Scotland trial showed no reduction in the rate of stroke-the risk after 5 years of follow-up was reported as 1.6 percent for both the pravastatin and placebo groups $(P=0.57)$, and a second cited study (the Air Force-Texas Coronary Atherosclerosis Prevention Study, or AFCAPS/ TexCAPS) did not list stroke as an individual end point. More to the point here, the 0.05 statistical difference reported by the authors using univariate analysis for pravastatin versus placebo was actually 0.10 upon application of multivariate analysis, arguably the more appropriate measure. ${ }^{39}$ Aside from the statistical debate, in which a small absolute difference may or may not be statistically "significant," there is the very important matter of practical significance. The study results suggested that it would require 750 patients to be treated for one year to prevent one nonfatal stroke, or more than $\$ 750,000$ in discounted drug cost, an unfavorable cost-effectiveness outcome and a very unfavorable cost outcome, compared to the risk reduction possible with aspirin. ${ }^{40}$ Previous research and a meta-analysis of clinical studies found no association between cholesterol levels and risk of stroke.

In this issue of the Journal, Arocho, Solis, Wade, Goldberg, and Tang discuss the very important subject of underlying characteristics of groups that might explain differences in the dependent measure found in study results. ${ }^{41}$ But within their analysis is the ever-present matter of practical versus statistical significance. As part of their findings of underlying differences among calcium channel blocker patients, they report $86 \%$ of amlodipine users had at least one professional service, compared to $84 \%$ of felodipine users $(P<0.05)$, in a study involving 17,667 patients. They also report a statistically significant $(P=0.0007)$ difference between average daily dose, $5.8 \mathrm{mg}$ for amlodipine patients versus $5.93 \mathrm{mg}$ for felodipine patients. They report average age but not median age and do not address the fact that $39.2 \%$ of amlodipine patients in their study were less than age 70 versus $33.9 \%$ of felodipine patients less than age 70 . Readers should note that this study was funded by the manufacturer of amlodipine. Managed care pharmacists would be interested in the formulary status of these two drugs at the time that the drug and medical claims were incurred in the health plans employed in the study, a fact not disclosed by the authors. Nevertheless, the authors are correct in highlighting the importance of managed care pharmacists investigating the underlying differences in patient populations, particularly in drug-todrug comparisons.

\section{Prevalence and Costs of Atopic Dermatitis}

Atopic dermatitis (AD), or eczema, is a chronic, relapsing inflammatory skin disease affecting about $10 \%$ of the Western population, with a prevalence estimate of $4 \%$ to $20 \%{ }^{42}$ The high range of the prevalence estimate is in children, and $80 \%$ to $90 \%$ of AD cases are diagnosed by age $5 .{ }^{43}$ The disease is physically distressing to the patient and often has an adverse effect on the quality of life. ${ }^{44}$ Fivenson, Arnold, Kaniecki, Cohen, Frech, and Finlay, in this issue of the Journal, provide estimates of some of the direct and indirect costs of $\mathrm{AD}$ among patients identified in one health plan, expressed in 1997 dollars ${ }^{45}$ Readers might note that about two thirds of the patients were cared for only by a dermatologist, suggesting that these patients were the more severe cases of $\mathrm{AD}$ in that population. Combined with the small prevalence, approximately $1.3 \%$ of plan members, the data suggest that this patient population is more representative of the moderate-to-severe population of $\mathrm{AD}$ patients. Yet, only 3 patients were rated as "severe" by provider-assessment. Nearly two thirds of the patients in this cohort were younger than 16 years, and $19.5 \%$ were younger than 4 years. There was one inpatient visit and no emergency room visits among 3,576 patientmonths in the study. The only inpatient visit occurred in a patient with a "mild" case of $\mathrm{AD}$, according to provider-assessed severity.

\section{בias Management-The Path to Better Evidence}

In this issue and on the Journal Web site (www.amcp.org) is a copy of the editorial policy, disclosure statement, and attestation required of all authors of articles published in JMCP, effective with the January/February 2002 issue. The detail is designed to obtain all disclosures necessary to permit readers to evaluate potential biases that may affect the objectives of the research, interpretation of data, conclusions, or other findings and to attribute to each listed author the specific contribution to the article.

Frederic R. Curtiss, PhD, RPh, CEBS, Editor-in-Chief 


\section{Editorial}

\section{REFERENCES}

1. Ruiz M, Torres A, Ewig S, et al. Noninvasive versus invasive microbial investigation in ventilator-associated pneumonia: evaluation of outcome. Am J Respir Crit Care Med. 2000;162(1):119-25.

2. Hepler CD. Regulating for outcomes as a systems response to the problem of drug-related morbidity. J Am Pharm Assoc. 2001;41(1)108-15.

3. Johnson JA, Bootman JL. Drug-related morbidity and mortality: A cost-ofillness model. Arch Intern Med. 1995;155:1949-56.

4. MacKinnon N, Hepler CD. Preventable drug-related morbidity in older adults: 1. Indicator development. J Managed Care Pharm. 2002;8(5): 365-71.

5. Morris C, Cantrill J. Preventable drug-related morbidity indicators in the U.S. and U.K. J Managed Care Pharm 2002; 8(5): 372-77.

6. Brennan TA, Leape LL, Laird NM, et al. Incidence of adverse events and negligence in hospitalized patients. New Engl J Med. 1991;324:370-76.

7. Leape LL, Woods DD, Hatlie MJ, et al. Promoting patient safety by preventing medical error. JAMA. 1998;280(16):1444-47.

8. Reason L. Human Error. Cambridge, England: Cambridge University Press; 1990.

9. History of the Joint Commission on Accreditation of Healthcare Organizations. Available at: http://www.jcaho.org/about+us/history/index.htm. Accessed August 21, 2002.

10. Traynor K. Patient safety standards focus on medical-error reduction, patient notification. Am J Health-Syst Pharm. 2001;August 1:1389.

11. In conversation with Sheldon Kotzin, Executive Editor, MEDLINE and Index Medicus, U.S. National Library of Medicine, Department of Health and Human Services; March 13, 2002.

12. James B. Making it easy to do it right. New Engl J Med. 2001;345(13):99192.

13. Shekelle P, Ortiz E, Rhodes S, et al. Validity of the Agency for Healthcare Research and Quality Clinical Practice Guidelines-how quickly do guidelines become outdated? JAMA. 2001;286:1461-67.

14. Martinez B. Doctors may lack data for treating certain ailments. Wall Street Journal. October 29, 2001:B8.

15. Latelines. Drug Top. 1998;January 5:7.

16. Bates DW, Leape LL, Cullen DJ, et al. Effect of computerized physician order entry and a team intervention on prevention of serious medication errors. JAMA. 1998;280:1311-16.

17. Landro L. FDA is urged to hasten efforts to require bar codes on drugs. Wall Street Journal. July 29, 2002:B5.

18. Moore JD. Getting the whole story-the way medication errors are reported affects the results. Mod Healthcare. 1998; December 21-8:46.

19. Dexter PR, Perkins S, Overhage JM, et al. A computerized reminder system to increase the use of preventive care for hospitalized patients, New Engl J Med. 2001;345(13):965-70.

20. James BC. Making it easy to do it right. New Engl J Med. 2001;345(13):991-92.

21. James BC. Quality improvement opportunities in health care-Making it Easy to do it Right. J Managed Care Pharm. 2002; 8(5):394-99.

22. Henry J. Kaiser Family Foundation. Medicare and prescription drugs. May 2001. Available at: http://www.kff.org.

23. White TJ, Chang E, Leslie S, et al. Patient adherence with HMG reductase inhibitor therapy among users of two types of prescription services. J Managed Care Pharm. 2002; 8(3):186-91.

24. Henry J. Kaiser Family Foundation; The Commonwealth Fund; Tufts-New England Medical Center. Seniors and prescription drugs-findings from a 2001 survey of seniors in 8 states. Available at: http://www.kff.org/content/2002/6049. Accessed August 5, 2002
25. Cox E, Henderson RR. Prescription-use behavior among Medicare beneficiaries with capped prescription benefits. J Managed Care Pharm. 2002; $8(5): 360-64$

26. Henry J. Kaiser Family Foundation. Trends and indicators in the changing health care marketplace, 2002. Chartbook. May 2002. Available at: www.kff.org/content/3161/. Accessed July 13, 2002.

27. Henry J. Kaiser Family Foundation. Uninsured in America: is health coverage adequate? Available at: www.kff.org/content/2002/4060/. Accessed July $13,2002$.

28. National Asssociation of Boards of Pharmacy. Survey of Pharmacy Law. 1997-1998; Park Ridge, IL.

29. The Script. California Board of Pharmacy. 2001; Oct:1,7

30. National Association of Boards of Pharmacy. Survey of Pharmacy Law. 2001-2002. Park Ridge, IL.

31. Two more states give pharmacists prescribing powers. Drug Top. 2000; May $15: 8$.

32. Latelines. Drug Top. 1999;May 3:10.

33. http://mlis.state.md.us/2002rs/billfile/HB0781.htm.

34. http://www.cga.state.ct.us/default.asp.

35. Gebhart F. When pharmacists have the final say on drug orders. Drug Top. 2002; April 15:HSE1,2.

36. Ravnan SL, Ravnan, MC, Manzo BA. Developing and implementing a pharmacist-managed, telephone-based Helicobacter pylori clinic. Am J HealthSyst Pharm. 2002;Apr 15(59):725-27.

37. Farris KB, Kumbera P, Halterman T, Fang G. Outcomes-based pharmacist reimbursement: reimbursing pharmacists for cognitive services. J Managed Care Pharm. 2002;8(5):383-93.

38. White HD, Simes RJ, Anderson NE et al. Pravastatin therapy and the risk of stroke. New Engl J Med. 2000;August 3:317-28.

39. Brett AS. Pravastatin therapy and the risk of stroke. New Engl J Med. 2000; December 21:1894.

40. Meilor JF, Utidehaag BMI. New Engl J Med. 2000;December 21:1895.

41. Arocho R, Solis A, Wade S, Goldberg G, Fang S. Exploring the methodological challenges of investigating comparison groups with different underlying characteristics: a case study. J Managed Care Pharm. 2002;8(5):353-59.

42. Knoell KA, Greer KE, Atopic dermatitis. Ped Rev. 1999;20:46-52.

43. Leung DYM. Disease management of atopic dermatitis: a practice parameter. Ann Allergy Asthma Immunol. 1997;79:197-211.

44. Lawson V, Lewis-Jones MS, Finaly AY, et al. The family impact of childhood atopic dermatitis: the Dermatitis Family Impact questionnaire. $\mathrm{Br} J$ Dermatol. 1998;138(1):107-13.

45. Fivenson D, Goldberg Arnold RJ, Kaniecki DJ, Cohen JL, Frech F, Finlay AY. The effect of atopic dermatitis on total burden of illness and quality of life on adults and children in a large managed care organization. J Managed Care Pharm. 2002;8(5):333-42

\section{Editor's Note:}

Mark McCoy, PharmD, MBA, Associate Director, Health Outcomes, Pharmacia Corporation, Peapack, New Jersey, was coauthor of the article "Relationship of Clinical Factors to the Use of Cox-2 Selective NSAIDs Within an Arthritis Population in a Large HMO" that appeared in the July/August 2002 issue of JMCP. 